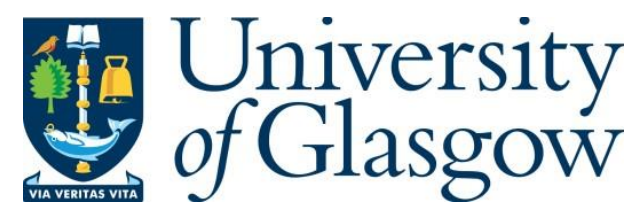

Hassib, M., Khamis, M., Schneegass, S., Shirazi, A. S. and Alt, F. (2016)

Investigating User Needs for Bio-sensing and Affective Wearables. In: CHI EA '16:

34th Annual ACM Conference Extended Abstracts on Human Factors in

Computing Systems, San Jose, CA, USA, 07-12 May 2016, pp. 1415-1422. ISBN

9781450340823.

There may be differences between this version and the published version. You are advised to consult the publisher's version if you wish to cite from it.

(C) The Authors 2016. This is the author's version of the work. It is posted here for your personal use. Not for redistribution. The definitive Version of Record was published in CHI EA '16: 34th Annual ACM Conference Extended Abstracts on Human Factors in Computing Systems, San Jose, CA, USA, 07-12 May 2016, pp. 1415-1422. ISBN 9781450340823 https://doi.org/10.1145/2851581.2892480.

\title{
http://eprints.gla.ac.uk/170229/
}

Deposited on: 5 October 2018

Enlighten - Research publications by members of the University of Glasgow http://eprints.gla.ac.uk 


\section{Investigating User Needs for Bio-sensing and Affective Wearables}

Mariam Hassib ${ }^{1,2}$, Mohamed Khamis ${ }^{1}$, Stefan Schneegass ${ }^{2}$, Alireza Sahami Shirazi ${ }^{3^{*}}$, Florian Alt ${ }^{1}$

${ }^{1}$ Media Informatics Group, University of Munich (LMU), Germany \{firstname.lastname\}@ifi.Imu.de

${ }^{2}$ VIS, University of Stuttgart, Germany \{firstname.lastname\}@vis.uni-stuttgart.de

${ }^{3}$ Yahoo Inc. CA, USA

alireza@yahoo-inc.com

"The majority of the work has been conducted while he was a researcher at the University of Stuttgart.
Permission to make digital or hard copies of part or all of this work for personal or classroom use is granted without fee provided that copies are not made or distributed for profit or commercial advantage and that copies bear this notice and the full citation on the first page. Copynights for third-party components of this work must be honored. CH'16 Exres, contact the Ow 2016, San Jose, CA, USA

ACM 978-1-4503-4082-3/16/05.

http $/ / /$ dx.doi.org/10.1145/2851581.2892480

\begin{abstract}
Bio-sensing wearables are currently advancing to provide users with a lot of information about their physiological and affective states. However, relatively little is known about users' interest in acquiring, sharing and receiving this information and through which channels and modalities. To close this gap, we report on the results of an online survey ( $\mathrm{N}=109$ ) exploring principle aspects of the design space of wearables such as data types, contexts, feedback modalities and sharing behaviors. Results show that users are interested in obtaining physiological, emotional and cognitive data through modalities beyond traditional touchscreen output. Valence of the information, whether positive or negative affects the sharing behaviors.
\end{abstract}

\section{Author Keywords}

physiological sensing; wearables; emotion; cognition

\section{ACM Classification Keywords}

H.5.3 [Information Interfaces and Presentation]: Group and Organization Interfaces; J.3. [Life and Medical Sciences]

\section{Introduction}

In recent years, myriads of smartphone applications and wearable devices (e.g., wristbands), have made physical activity tracking, such as step counting and sleep monitoring, a ubiquitous activity. Sensors are becoming smaller 


\begin{tabular}{l}
\hline Physiological \\
\hline Heart Rate (HR) \\
Blood Pressure (BP) \\
Body Temperature (BT) \\
Breathing Rate (BR) \\
Electrodermal Activity (EDA) \\
Brain Signals(EEG) \\
\hline Emotional \\
\hline Happiness \\
Anger \\
Sadness \\
Boredom \\
Excitement \\
Fear \\
Surprise \\
\hline Cognitive \\
\hline Stress \\
ConcentrationAttention \\
RelaxationMeditation \\
\hline
\end{tabular}

Table 2: List of 16 types of information included in the survey.

\begin{tabular}{|c|c|c|}
\hline & Device & Data Provided \\
\hline 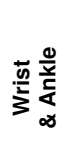 & $\begin{array}{l}\text { Apple Watch, Samsung Gear, Garmin, } \\
\text { FitBit, Jawbone, LG Watch } \\
\text { Embrace, Affectiva Q Sensor } \\
\text { Zensorium Being }\end{array}$ & $\begin{array}{l}\text { Activity tracking, HR } \\
\text { Stress, EDA } \\
\text { Stress, calm, excitement, HR } \\
\text { \& BP }\end{array}$ \\
\hline 『 & $\begin{array}{l}\text { Neurosky Mindwave \& Mindset } \\
\text { Emotiv EPOC/Insight } \\
\text { Melon } \\
\text { Melomind }\end{array}$ & $\begin{array}{l}\text { EEG, attention \& meditation } \\
\text { EEG, attention, relaxation, } \\
\text { boredom \& engagement } \\
\text { EEG, concentration, calm } \\
\text { EEG, stress, relaxation }\end{array}$ \\
\hline 궁 & $\begin{array}{l}\text { OM Signal Shirt } \\
\text { Spire } \\
\text { BioHarness } \\
\text { TempTraq }\end{array}$ & $\begin{array}{l}\text { HR, BR } \\
\text { Calm \& focus, BR } \\
\text { HR, BR } \\
\text { BT }\end{array}$ \\
\hline
\end{tabular}

Table 1: Overview of commercial wearables in the market offering physiological or affective information.

and more powerful, making additional information, such as physiological data (e.g., heart/breathing rates) as well as user's emotions and mental state available. Table 1 provides an overview of commercial biosensing and affective wearables. As a result, the application areas of affective [13] or biosensing wearables expand from medical monitoring to personal information applications (cf., Quantified Self ${ }^{1}$ movement).

Research has taken up on this development and focused on exploiting wearables to extract accurate information from the body's raw signals to provide value for users $[1,6,8]$. The interconnection between wearable trackers and smartphones allows collected data to be shared with third party applications and groups of people, e.g., through social media. Researchers focused on exploring the effects of sharing such private information with different groups of people $[3,7,9,14,16]$. Additionally, researchers explored user preferences for using wearables in different contexts [2, 11]. While the design space of biometric and affective wearables

\footnotetext{
${ }^{1}$ http://quantifiedself.com/
}

includes many dimensions, we chose to focus in this part of our research on identifying potential user needs with regard to aspects of utility, connectivity, and feedback of the new biometric and affective wearables. We aim to understand user interest in acquiring and sharing biometric and emotional information, as well as exploring new channels and modalities for presenting this information. We report the quantitative and qualitative results of an online survey and discuss insights arising from our results that can help wearable designers form an understanding of their users.

\section{Survey}

We constructed an online survey, consisting of three main sections, to discover user needs:

- Acquiring data about oneself

- Sharing data with others

- Receiving data from others

The survey started with demographic information: age, country of origin, level of education and current profession. We asked participants to indicate the number of minutes they spend on average on social media per day and if they own wearable tracking devices or activity tracking apps.

\section{Acquiring Data about Oneself}

After a thorough investigation of related literature and current affective and biometric wearables in the consumer market, we augmented a list of 16 information types divided into three main categories: raw physiological, emotional, and cognitive data (see Table 2). Participants were asked to rate their interest in acquiring each type of information on a 5point Likert scale ( 1 =strongly disagree, $5=$ strongly agree). We gave participants use cases describing a wearable wristband that shows their heart rate or a ring that changes color to indicate their current mood. 


\begin{tabular}{l}
\hline Channels \\
\hline Smartphone \\
Wearable Gadget \\
Website \\
Social Media \\
Ambient Display \\
\hline Modalities \\
\hline Visual \\
Auditory \\
Haptic (Vibro-tactile) \\
Olefactory (Smell) \\
\hline
\end{tabular}

Table 3: List of the identified channels and modalities for acquiring, sharing and receiving information.
We presented participants with several channels and modalities for getting information based on existing channels and modalities researched or used in market-available wearables and asked participants to choose their preferred ones (depicted in Table 3). Finally, we asked participants to provide past situations in which knowing biometric or affective information about themselves could have been helpful.

\section{Sharing Data with Others}

Based on prior literature [4, 9], we presented participants six categories of people with whom they can share information: no one, partner, family, close friends, colleagues, and public, and asked them to select, for each type of information, one or more categories with whom they would like to share the information. Then we asked the participants to choose the preferred sharing channel(s) per type of information (physiological, emotional, cognitive) (Table 3).

Additionally, we asked three open ended questions: (1) if they already share their emotions or state, through which channel and for what reason. (2) They should express their feelings upon sharing information and (3) mention situations in which they deliberately decided not to share.

\section{Receiving Data from Others}

In the next part, we asked participants to select categories of people whom they would like to receive information from. We then asked about the channel(s) and modality(s) preferred for receiving this information. Finally, in an open ended question we asked for a specific example of how they reacted upon receiving information from others.

\section{Recruitment}

We distributed the survey, which took approximately $20 \mathrm{~min}$ utes, through university mailing lists and university social media groups. Participants who completed the survey took part in a lottery for three 20 Euros Amazon gift vouchers.

\section{Results}

In total 210 people attempted the survey while $109 \mathrm{com}$ pleted it fully (52 female, aged 19 to $38, M=25.19, S D=3.99$ ). Quantitative results are based on the fully completed survey while qualitative insights are drawn from all participants. Participants included university students (17), with bachelor (42), or graduate (22) degrees. Professions included architects, pharmacists, engineers, doctors, marketeers, sociologists, and bankers. They came from 19 different countries from 5 continents. All survey answers were in English. All participants owned smartphones and spend at least one hour using social media per day. Ten own wearable activity trackers and 38 participants use activity tracking apps.

Interest in Information Types

Participants ranked their interest in obtaining 16 types of information from a wearable gadget. In physiological data, heart rate, blood pressure, body temperature, and breathing rate are most interesting for the users with a median of "Agree" ( $M e d=3.5)$. Scores of emotional information, except for happiness and anger, are lower ( $M e d=3)$. Finally, interest in learning about their cognitive state is highest

$(M e d=4)$. Comparing the three categories of data, we conducted a Wilcoxon-sign ranked test that shows that participants interest in obtaining cognitive information is significantly higher than physiological $(Z=-2.078, p<.05)$ as well as emotional information $(Z=-5.281, p<.05)$.

Feedback Channels and Modalities

Smartphones, being ubiquitous and private, were the participants' top preference to receive all types of data $(73 \%)$, compared to $43 \%$ for receiving information through the wearable gadget itself, $25 \%$ through an ambient display, $12 \%$ through a website and only $3 \%$ through social media.

Users expect visual representations of information. There is no clear influence of the type of information on the modality. 


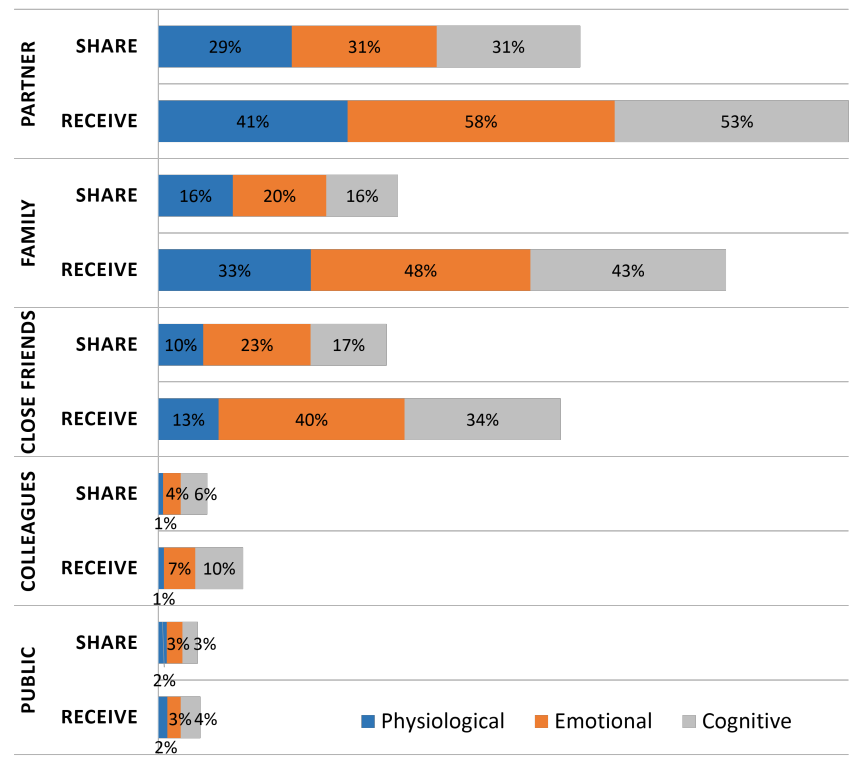

Figure 1: Comparison of sharing and receiving different types of information with different categories of people. In general

participants want to receive more than share information. Interest in sharing with and receiving emotional and cognitive state from the partner is highest.

The only difference we discovered was with regard to scent as a new modality. Here, participants seemed more prone to choose this modality for emotional data $(10 \%)$.

Sharing and Receiving Information

We found similar preferences across categories of people - users would mostly share with partners and family members. Figure 1 depicts the users' preferences with regard to sharing and receiving different types of information. Cognitive attention, relaxation, and stress sharing was popular with different categories. Sharing stress, being a negative state, with partners and close friends was more popular than sharing it with family members.

In general, participants want to receive more information than they want to share (Figure 1). We can see that participants would mostly like to receive physiological data from their partner and friends. Additionally they are interested to know the emotional and mental state of family members.

\section{Qualitative Findings}

We gathered qualitative data through the open-ended questions previously introduced. Three researchers conducted a full data walkthrough and coded the answers into different themes (cf., Figure 2). The created themes were iterated on until an agreement was reached for each question.

Context of Use for Biometric and Affective Wearables From 119 different responses (also considering partially filled responses), we identified 11 main contexts in which participants mentioned they would need to acquire biometric, cognitive, or emotional information about themselves. While $21 \%$ of the answers concern Sports and $24 \%$ concern Health, participants suggested a multitude of other contexts.

Many participants mentioned that they would like to know their physiological and cognitive state in stressful situations (e.g., conflicts). In context of workplace, P216 stated that he would use this information to defend himself in front of his boss. Others stated they would want such information during meetings or after a long day of study. Several participants mentioned that knowing their high concentration times would help schedule their work and breaks. Whereas one participant stated that physiological sensors can help in decision making "When making a decision, Maybe the measured information of the body can help [me] making the right decision." (P141) Others (9\%) saw no value in know- 


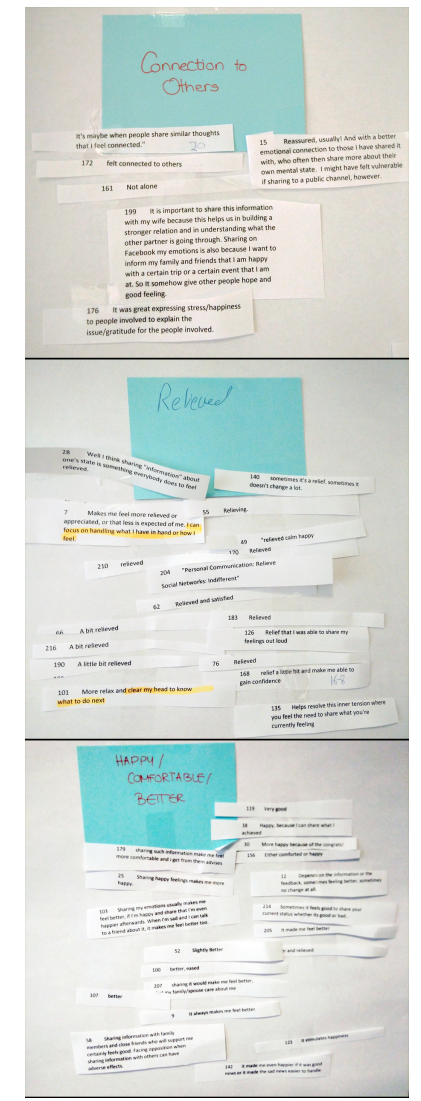

Figure 2: Three of the themes extracted from the data walkthrough process of the answers to: According to the situation you earlier described in which you shared emotional and cognitive information with others, how did that make you feel afterwards? ing this information and stated that one should be more self aware.

\section{Sharing Experiences and Effects}

Participants had diverse experiences in sharing physiological and emotional data. The valence of the data, whether it is a positive or negative emotion or state, strongly affected the sharing behaviors. Many participants stated that they would share their physiological information (such as blood pressure) with family members in case they are in trouble. However they would not share negative emotions, so as not to worry them. The motivations for sharing are various. Sharing to seek comfort during a hardship or sickness, to increase motivation at work or while studying, and sharing concentration not to be interrupted, were among the reasons stated by participants.

Participants share information through private texting (e.g., whatsapp), face-to-face conversations, calls, and social media. Results show that sharing publicly is often associated with positive achievements or feelings: "Sharing my happiness on social media after the defense of my Master thesis " (P30). "When I am happy because I have gotten something that I wished" (P119). Sharing negative states is done through private forms of communication. Many participants reported that they share negative feelings for venting out rather than for expecting particular feedback.

After sharing emotional, physiological or cognitive information, participants stated they felt calm, relieved, motivated, enthusiastic, more confident, and generally better. Six participants stated they feel more connected to people receiving shared information and their relationship is strengthened. However, sharing may have adverse effects on sharers in case of not receiving adequate feedback [5]: P12: "Depends on the information or feedback, sometimes feeling better, sometimes no change at all." Some partic- ipants stated feeling exposed or vulnerable when sharing through particular channels (e.g., Facebook) or when not receiving the expected feedback (P15, P58).

\section{Reasons not to Share}

We followed the same data walkthrough process explained earlier to code the answers of participants into five themes that were each mentioned by at least two participants.

Privacy and Lack of Trust: 29 participants mentioned privacy concerns as main reasons for not sharing. For example, P201 stated that: "Sharing my feelings with the public is nonsense, it allows people to intrude in your life."

Lack of Reason: 15 participants stated there is no reason to share information with others as "situations won't change"(P126); they did not see real value in sharing their emotions with others.

Consideration for Others: Ten participants mentioned that they would not share negative information with others so as not to worry them. "I don't often speak about being sad, angry, stressed with some family or friends. This is because I know they will be affected." (P27). "When I know that the information would have a negative impact on my family or spouse as they would worry about me, I don't share." (P140). Others stated they would not share positive information in case the receiver was in a contrasting situation.

Fear of Rejection and Judgment: Several participants stated they do not share their emotions in fear of embarrassment or judgment. "Sharing negative emotions is tricky because it can create rejection or judging." (P49). Many answered that they will not share to preserve their personal image and for fear of negative self representation. For example, P216 would not share any information whenever it 
would make her look incompetent. P7 would not share feelings he is ashamed of especially if deemed pitiful.

Self Discipline: Two participants stated not to share emotions/mental states with others as a form of self discipline. "I teach myself not to share everything about oneself" (P172).

\section{Discussion}

New Visualization Channels and Modalities

Although current wearables provide visual feedback through smartphone apps and LEDs, results show that potential users are also interested in novel or subtle feedback. 30\% of participants are interested in acquiring physiological information about themselves via tactile feedback. Feedback channels should request attention instead of demanding it. Hence the concept of feedback in-the-world and beyond the touchscreen via ambient displays, haptic or olfactory feedback could be possible methods to achieve that. Our results emphasize exploring further feedback channels and modalities. Recent methods, such as electrical muscle stimulation (EMS) [12] or air-based feedback $[15,10]$ expand the range of messages that can be delivered.

Context vs. People Categories in Sharing

Current wearables provide users the option to share data with predefined or user-defined categories of people. However, one of the insights we gained from the qualitative results is that context of sharing and the valence of data to be shared play a crucial role in sharing behaviors. Data with negative valence, whether emotional or physiological, is less likely to be shared with closer people. On the other hand, data with negative valence sometimes is indeed a cause of sharing for some people since it induces relief and support from others.

Designers have to account for the type of information and the context of sharing. System-sided recommendations can be used to instruct the user about cases when the sharedwith person is in an opposing mental or emotional state

(e.g., based on biosignal data). Recommendations can also suggest whom to share with based on personal data via learning from behavior. This can help encourage mutual sharing and foster better relationships.

\section{Encouraging Mutual Sharing}

Figure 1 shows that users are more interested in receiving information than they are willing to share themselves. This is the case for all types of information. This suggests that designers should consider ways of encouraging mutual sharing of information. One way is to enforce a reciprocation system; for example, a user can learn about the partner's physiological state only if the user decided to share own information.

\section{Conclusion and Future Work}

In this research we presented the results of an online survey $(n=109)$ on bio-sensing and affective wearables. We discerned the users' needs and expectations in terms of the utility of the wearable, the provided feedback channels and modalities and the possible connectivity and sharing features. We analyzed and discussed results of the survey from these dimensions and provided several useful insights for the $\mathrm{HCl}$ community.

We believe our work can help designers understand potential users and create valuable applications for biometric and affective wearables. In the future we will build upon our findings to delve deeper into each design aspect and conduct focus groups and design workshops to put the findings of the survey into the practical world. 


\section{Acknowledgments}

The research leading to these results has received funding from the European Union Seventh Framework Programme ([FP7/2007-2013]) under grant agreement number 323849.

\section{References}

[1] Yadid Ayzenberg, Javier Hernandez Rivera, and Rosalind Picard. 2012. FEEL: Frequent EDA and Event Logging - a Mobile Social Interaction Stress Monitoring System. In CHI '12 Extended Abstracts on Human Factors in Computing Systems (CHI EA '12). ACM, New York, NY, USA, 2357-2362. DOI : http://dx.doi.org/10.1145/2212776.2223802

[2] Debjanee Barua, Judy Kay, and Cécile Paris. 2013. Viewing and controlling personal sensor data: what do users want? In Persuasive Technology. Springer, 15-26.

[3] Franco Curmi, Maria Angela Ferrario, Jen Southern, and Jon Whittle. 2013. HeartLink: Open Broadcast of Live Biometric Data to Social Networks. In Proceedings of the SIGCHI Conference on Human Factors in Computing Systems (CHI '13). ACM, New York, NY, USA, 1749-1758. DOI : http://dx.doi.org/10.1145/ 2470654.2466231

[4] Franco Curmi, Maria Angela Ferrario, and Jon Whittle. 2014. Sharing Real-time Biometric Data Across Social Networks: Requirements for Research Experiments. In Proceedings of the 2014 Conference on Designing Interactive Systems (DIS '14). ACM, New York, NY, USA, 657-666. DOI : http://dx.doi.org/10.1145/2598510. 2598515

[5] Daniel A. Epstein, Bradley H. Jacobson, Elizabeth
Bales, David W. McDonald, and Sean A. Munson. 2015. From "Nobody Cares" to "Way to Go!": A Design Framework for Social Sharing in Personal Informatics. In Proceedings of the 18th ACM Conference on Computer Supported Cooperative Work \&\#38; Social Computing (CSCW '15). ACM, New York, NY, USA, 16221636. DOI : http://dx.doi.org/10.1145/2675133.2675135

[6] J. Hernandez, Yin Li, J.M. Rehg, and R.W. Picard. 2014. BioGlass: Physiological parameter estimation using a head-mounted wearable device. In Wireless Mobile Communication and Healthcare (Mobihealth), 2014 EAl 4th International Conference on. 55-58. DOI : http://dx.doi.org/10.1109/MOBIHEALTH.2014.7015908

[7] Rohit Ashok Khot, Jeewon Lee, Deepti Aggarwal, Larissa Hjorth, and Florian 'Floyd' Mueller. 2015. TastyBeats: Designing Palatable Representations of Physical Activity. In Proceedings of the 33rd Annual ACM Conference on Human Factors in Computing Systems (CHI '15). ACM, New York, NY, USA, 29332942. DOI : http://dx.doi.org/10.1145/2702123.2702197

[8] Diana MacLean, Asta Roseway, and Mary Czerwinski. 2013. MoodWings: A Wearable Biofeedback Device for Real-time Stress Intervention. In Proceedings of the 6th International Conference on PErvasive Technologies Related to Assistive Environments (PETRA '13). ACM, New York, NY, USA, Article 66, 8 pages. DOI : http://dx.doi.org/10.1145/2504335.2504406

[9] Hyeryung Christine Min and Tek-Jin Nam. 2014. Biosignal Sharing for Affective Connectedness. In $\mathrm{CHI}$ '14 Extended Abstracts on Human Factors in Computing Systems (CHI EA '14). ACM, New York, NY, USA, 2191-2196. DOI : http://dx.doi.org/10.1145/2559206. 2581345 
[10] Omar Mowafi, Mohamed Khamis, and Wael Abouelsaadat. 2015. AirDisplay: Experimenting with Air Flow as a Communication Medium. In Human-Computer Interaction - INTERACT 2015, Julio Abascal, Simone Barbosa, Mirko Fetter, Tom Gross, Philippe Palanque, and Marco Winckler (Eds.). Lecture Notes in Computer Science, Vol. 9296. Springer International Publishing, 316-323. DOI : http://dx.doi.org/10. 1007/978-3-319-22701-6 23

[11] Misha Patel and Aisling Ann O'Kane. 2015. Contextual Influences on the Use and Non-Use of Digital Technology While Exercising at the Gym. In Proceedings of the 33rd Annual ACM Conference on Human Factors in Computing Systems (CHI '15). ACM, New York, NY, USA, 2923-2932. DOI : http: //dx.doi.org/10.1145/2702123.2702384

[12] Max Pfeiffer, Tim Dünte, Stefan Schneegass, Florian Alt, and Michael Rohs. 2015. Cruise Control for Pedestrians: Controlling Walking Direction Using Electrical Muscle Stimulation. In Proceedings of the 33rd Annual ACM Conference on Human Factors in Computing Systems (CHI '15). ACM, New York, NY, USA, 2505-2514. DOI : http://dx.doi.org/10.1145/2702123.
2702190

[13] R.W. Picard and J. Healey. 1997. Affective wearables. Personal Technologies 1, 4 (1997), 231-240. DOI : http://dx.doi.org/10.1007/BF01682026

[14] Petr Slovák, Joris Janssen, and Geraldine Fitzpatrick. 2012. Understanding Heart Rate Sharing: Towards Unpacking Physiosocial Space. In Proceedings of the SIGCHI Conference on Human Factors in Computing Systems (CHI '12). ACM, New York, NY, USA, 859868. DOI : http://dx.doi.org/10.1145/2207676.2208526

[15] Rajinder Sodhi, Ivan Poupyrev, Matthew Glisson, and Ali Israr. 2013. AIREAL: Interactive Tactile Experiences in Free Air. ACM Trans. Graph. 32, 4, Article 134 (July 2013), 10 pages. DOI : http://dx.doi.org/10.1145/2461912. 2462007

[16] Wouter Walmink, Danielle Wilde, and Florian 'Floyd' Mueller. 2013. Displaying Heart Rate Data on a Bicycle Helmet to Support Social Exertion Experiences. In Proceedings of the 8th International Conference on Tangible, Embedded and Embodied Interaction (TEI '14). ACM, New York, NY, USA, 97-104. DOI : http://dx.doi.org/10.1145/2540930.2540970 